\title{
A influência do isolamento social e do COVID-19 em atletas de alto rendimento
}

\author{
The influence of social isolation and COVID-19 on high-performance athletes \\ La influencia del aislamiento social y del COVID-19 en atletas de alto rendimiento
}

Recebido: 01/02/2022 | Revisado: 14/02/2022 | Aceito: 23/02/2022 | Publicado: 04/03/2022

\author{
Lucas Vinicius da Costa Lima \\ ORCID: https://orcid.org/0000-0002-1987-6294 \\ Universidade Católica de Pernambuco, Brasil \\ E-mail: lucasfisiolima@outlook.com \\ Bruno Gilberto de Melo e Silva \\ ORCID: https://orcid.org/0000-0002-2946-3119 \\ Universidade Católica de Pernambuco, Brasil \\ E-mail: bruno.melo16@unicap.br \\ Vinicius Alexandre Alves da Silva \\ ORCID: https://orcid.org/0000-0001-8938-2882 \\ Universidade Católica de Pernambuco, Brasil \\ E-mail: alexandrevinicius785@gmail.com \\ Juliana Bezerra Vieira Costa Cavalcanti \\ ORCID: https://orcid.org/0000-0003-4628-7759 \\ Universidade Católica de Pernambuco, Brasil \\ E-mail: julianabvcc@gmail.com \\ Ingrid Holanda Silva \\ ORCID: https://orcid.org/0000-0003-3374-6354 \\ Universidade Católica de Pernambuco, Brasil \\ E-mail: ingridholanda11@outlook.com \\ Gabriel Michiles de Assunção Santos \\ ORCID: https://orcid.org/0000-0003-2717-9381 \\ Universidade Católica de Pernambuco, Brasil \\ E-mail: bielmichiles@gmail.com \\ Natasha de Oliveira Vila Nova \\ ORCID: https://orcid.org/0000-0002-4630-5809 \\ Universidade Católica de Pernambuco, Brasil \\ E-mail: natsha.vn@icloud.com \\ João Vitor Seara Machado \\ ORCID: https://orcid.org/0000-0003-1206-053X \\ Universidade Católica de Pernambuco, Brasil \\ E-mail: vitor.jvsm@hotmail.com
}

\begin{abstract}
Resumo
O presente estudo tem como objetivo analisar a influência do período de isolamento social e do contágio do Covid nos treinos, ritmo, desempenho e lesões em atletas de alto rendimento. Trata-se de um estudo observacional, descritivo, de corte transversal e quantitativo. Em que foram incluídos no estudo atletas que estivessem ligados a alguma federação e de ambos os sexos, que finalizasse adequadamente o formulário. Os resultados identificaram que dos 37 atletas avaliados, 62,2\% retornaram fora de ritmo e com condicionamento físico baixo após isolamento e que 64,9\% não sofreram nenhuma lesão ao retorno dos treinos. Em relação ao diagnóstico confirmado de COVID-19, 21,6\% jogadores foram infectados, destes, 50\% afirmaram que o COVID-19 influenciou moderadamente, precisando de um período de mais de um mês para retornar ao desempenho e ritmo anterior a infecção. Diante do exposto, as considerações finais do estudo demonstram que a presente pesquisa não revelou evidências causais sobre o impacto do isolamento social e do contágio do COVID-19 em relação com o número ou risco de lesões. Contudo, foi evidenciado algumas influências no desempenho, ritmo e condicionamento físico.
\end{abstract}

Palavras-chave: Coronavírus; Distanciamento social; Atletas de elite; Lesão.

\begin{abstract}
Objective: To analyze the influence of Covid's isolation period and contagion on training, pace, performance and injuries in high-performance athletes. Methodology: This is an observational, descriptive, cross-sectional and quantitative study. Inclusion criteria: athletes who were linked to a federation and of both sexes. Exclusion athletes were athletes who did not respond adequately to the form. A form was made with the athletes about their socioclinical data and questions related to Covid-19, isolation, return to training, performance and injuries, after which a descriptive analysis of the collected data was carried out. Results: The sample was formed by a total of 37 athletes, $62.2 \%$ returned out of pace and with low physical conditioning after isolation; $64.9 \%$ did not suffer any injuries when
\end{abstract}


returning from training. Regarding the confirmed diagnosis of COVID-19, 21.6\% of players were infected, of which $50 \%$ stated that COVID-19 had a moderate influence, needing a period of more than a month to return to the performance and pace of before. Final considerations: The present study did not reveal any causal evidence about the impact of the isolation and contagion of COVID-19 in relation to the number or risk of injuries. However, some influences on performance, rhythm and physical conditioning were evidenced.

Keywords: Coronavirus; Social distancing; Elite athletes; Lesion.

\section{Resumen}

El presente estudio tiene por objetivo analizar la influencia del periodo del aislamiento social y del contagio del Covid en los entrenamientos, ritmo, desempeño y lesiones en atletas de alto rendimiento. Se trata de un estudio observacional, descriptivo, transversal y cuantitativo. Fueron incluidos en lo estudio atletas vinculados a alguna federación y de ambos sexos, los cuales hayan completado el formulario. Los resultados identificaron que, de los 37 atletas evaluados, 62,2\% regresaron fuera de ritmo y con bajo acondicionamiento físico post-aislamiento y que 64,9\% no sufrieron ninguna lesión al regresar a los entrenamientos. En relación al diagnóstico confirmado de COVID-19, 21,6\% jugadores fueron infectados, de los cuales 50\% afirmaron que el COVID-19 influenció moderadamente, necesitando de un periodo mayor que un mes para volver a su desempeño y ritmo anteriores a la infección. Frente a lo expuesto, las consideraciones finales del estudio demostraron que la presente pesquisa no reveló evidencias causales sobre el impacto del aislamiento social y del contagio del COVID-19 en relación al número o al riesgo de lesiones. Sin embargo, fueron evidenciadas algunas influencias en el desempeño, ritmo y acondicionamiento físico.

Palabras clave: Coronavirus; Aislamiento social; Atleta de elite; Lesión.

\section{Introdução}

No final de dezembro de 2019, um grupo de pacientes foi admitido em hospitais com diagnóstico inicial de pneumonia de etiologia desconhecida. Esses pacientes estavam epidemiologicamente ligados a um mercado atacadista de frutos do mar e animais úmidos em Wuhan, província de Hubei, China. Sendo posteriormente, nomeada e identificada como a doença do coronavírus (COVID-19), causada pelo agente SARS-COV2, a qual demostrou ser uma doença potencialmente fatal, promovendo assim, grande preocupação para a saúde pública global. Uma vez que COVID-19 causa síndrome respiratória, podendo ser aguda ou grave, potencialmente fatal, por possuir um caráter sistêmico, o que possibilita o desenvolvimento de comorbidades (Brooks et al, 2020; Shaoqing et al, 2020).

Os sintomas da infecção por COVID-19 aparecem após um intervalo de incubação de aproximadamente de 2 a 5 dias. O período desde o início dos sintomas de COVID-19 até a morte variou de 6 a 41 dias, com uma mediana de 14 dias. Esse período depende da idade do paciente e do estado de seu sistema imunológico. Foi mais curto entre pacientes $>70$ anos quando comparados com os indivíduos menores de 70 anos (Guan et al, 2020). Os sintomas mais comuns no início da doença COVID-19 são febre, tosse e fadiga, enquanto outros sintomas incluem produção de expectoração, dor de cabeça, hemoptise, diarreia, dispneia, linfopenia, ausência de paladar e olfato. As características clínicas reveladas na tomografia computadorizada de tórax apresentam o quadro de pneumonia, com incidência de opacidades em vidro fosco. Além dessas situações, o desenvolvimento de síndrome do desconforto respiratório agudo, lesão cardíaca favorecem o desfecho de mortalidade (Sepehrinezhad et al, 2020).

Devido à alta capacidade de propagação do vírus de pessoa a pessoa, uma das soluções globais foram a contenção das populações em seus lares, que posteriormente submetidos a medidas ainda mais extensivas, a fim proteger e reduzir a transmissão do vírus, e assim controlar o surto. Sendo assim, todas as principais ligas e torneios esportivos foram suspensos ou cancelados devido ao COVID-19 desde o início de março de 2020, com as paralizações das atividades dos clubes, por mais que o atleta típico possa sentir apenas sintomas leves como resultado do COVID-19, estratégias de prevenção são necessárias para reduzir a transmissão e o risco de propagação do corona vírus para indivíduos dentro de uma comunidade que estão em maior risco de grave infecção ou morte, que inclui pessoas mais velhas e imunocomprometidas (Yu et al, 2020; Bai et al, 2020). 
Os atletas de alto rendimento que trabalham com o esporte, precisam produzir renda para sobreviver no meio social, sendo assim, necessitam de grande dedicação, reponsabilidade e cuidados com o condicionamento, performance, alimentação e o próprio psicológico. (Nahshon et al, 2020). Os atletas necessitaram ser afastados dos clubes e federações nos momentos mais críticos da pandemia. Para manter a aptidão cardiovascular foi recomendado que os atletas treinassem durante o período de isolamento em casa, dependendo do equipamento disponível, podendo incluir a bicicleta ergométrica, esteira e treinamento de resistência para auxiliar os treinos, com orientação e monitoramento de profissionais como fisioterapeuta, fisiologista e/ou preparador físico que passassem treinos de força e condicionamento ou até mesmo exercícios a serem feitos com fisiologista de forma remota (Barker-Davies et al, 2020).

Como o número de infecções e as hospitalizações diminuíram, o bloqueio restrito foi gradualmente diluído, os esportes em equipe começaram a reintroduzir seu treinamento de rotinas em grupos, e a Bundesliga (liga de futebol alemã) foi umas das pioneiras a ser reativada, iniciando as atividades e treinos da liga profissional, na qual ficou com portas fechadas em 16 de maio de 2020 e seguiu todos os protocolos de segurança emitidos, com exames regulares, tempo de treino, divisão de grupos reduzidos, materiais de proteção, higienização e implementação de verificações de temperatura diária ${ }^{11,12}$. Dessa forma, considerando que a pandemia do Sars-Cov-2 há grandes influências para a população de modo geral e em diferentes aspectos da saúde, se faz necessário realizar estudos que analise as influências do isolamento social e do contágio do COVID-19 em atletas profissionais em diferentes aspectos da saúde.

\section{Metodologia}

O estudo é do tipo observacional, descritivo, de corte transversal e de caráter quantitativo. As coletas de dados foram realizadas através de um formulário eletrônico no período de setembro a novembro de 2020. A presente pesquisa está vinculada a Universidade Católica de Pernambuco a escola de saúde e ciências da vida e ao projeto de pesquisa intitulado em "Avaliação, prevenção e tratamento fisioterapêutico das lesões relacionadas ao esporte" com número de CAAE: 10321419.4.0000.5206, aprovado pelo comitê de ética e pesquisas com seres humanos com número de protocolo: 3.234.043, pertencente ao grupo de fisioterapia baseada em evidência. A amostra foi por conveniência de atletas de alto rendimento que aceitassem participar do estudo, sendo incluídos na pesquisa de ambos os sexos, ligados a um clube e ou a uma federação e que tivessem assinado o termo de consentimento livre esclarecido (TCLE). E excluídos, os atletas que não responderam adequadamente o formulário ou não faziam parte de uma federação.

Foi realizada uma conversa através do dispositivo móvel, para expor aos indivíduos os objetivos do estudo, os pontos que seriam avaliados e como seria realizada a coleta de informações. Após a sua concordância para participar da pesquisa, foi encaminhado via e-mail ou por anexo no Whatsapp ${ }^{\circledR}$, do atleta para que fosse assinado o TCLE. Posteriormente a assinatura do termo, iniciamos a coleta de dados, com utilização de um questionário construído pelos autores da pesquisa, para a obtenção do perfil sócio clínico, com dados referentes a sexo, idade, qual esporte atua e por qual clube ou federação.

Após avaliar o perfil sócio clínico, os atletas foram submetidos a perguntas sobre o isolamento social no período de maior calamidade da pandemia, onde se encontrava todos esportes e clubes com atividades paralisadas, foram questionados sobre o quanto esse período influenciou o retorno dos jogadores aos treinos e atividades do clube ou federação em relação ao seu desempenho, ritmo, condicionamento físico e lesões. Seguindo o questionário, agora os atletas foram perguntados se tiveram contágio com o COVID-19 com diagnóstico confirmado, após isto, a pesquisa e as perguntas foram direcionadas somente para aqueles que foram infectados pelo vírus, os que não possuíram o corona vírus, ficaram isentos de responder as próximas perguntas, os questionamentos a seguir foram relacionadas ao retorno do período de 15 dias de quarentena do COVID-19 com o desempenho, ritmo, condicionamento físico e lesões dos atletas contagiados. 


\section{Resultados}

A amostra em estudo foi composta por 37 atletas de alto rendimento, sendo 28 (75,7\%) do sexo masculino e 9 $(24,3 \%)$ do sexo feminino, praticantes de diferentes esportes, 24 (64.9\%) atletas de futebol, 11 (29,7\%) de handebol e 2 $(5,4 \%)$ de voleibol. Dentro dessa perspectiva, a amostra distribui-se em diversos clubes, obtendo $12(32,4 \%)$ atletas do Sport Clube do Recife, 7 (18,9\%) do Clube Náutico Capibaribe, 6 (16,2\%) do Santa Cruz Futebol Clube, 5 (13,5\%) Salgueiro Atlético Clube, 4 (10.8\%) Vera Cruz F.C, 2 (5,4\%) Pesqueira F.C e 1 (2,7\%) do Portimonense F.C.

Ao analisarmos as respostas dos atletas, quando perguntados se no período de maior isolamento, você treinou em casa de alguma forma adaptativa, ou em um local reservado, 35 (94,6\%) dos jogadores responderam que treinou e $2(5,4 \%)$ responderam que não treinou nesse tempo de isolamento total. A tabela 1, por sua vez, mostra o quanto o isolamento influenciou na volta aos treinos, observamos que sua grande maioria retornou aos treinos com condicionamento físico baixo e um pouco fora de ritmo. Após isto, com a reabertura dos clubes pós-isolamento, e volta dos treinos oficialmente, foram questionados se tiveram alguma lesão, caso a resposta fosse sim, relataram qual foi a lesão. Na sua maioria, 24 (64,9\%) jogadores não sofreram nenhuma lesão e 13(35,1\%) tiveram lesões, no qual as lesões musculares foi a mais acometida, ocorrendo em 4 (30,7\%) jogadores, seguido do rompimento do ligamento cruzado anterior em 3 (23,1\%) jogadores, obtevese também $2(15,4 \%)$ atletas com lesão na articulação glenoumeral, 2 (15,4\%) com fratura de platô tibial, 1 (7,69\%) com lesão de menisco e 1 (7,69\%) com avulsão do ilíaco.

Além disso, os atletas foram questionados se tiveram diagnóstico confirmado de COVID-19, obteve-se que 29 (78.4\%) jogadores não teve contato com o vírus e que uma pequena parcela foi contagiada no total de $8(21,6 \%)$ jogadores, a partir disso, a pesquisa prosseguiu somente com a amostra que testaram positivo no exame para corona vírus. Em relação aos sintomas apresentados 50\% da amostra teve febre, ausência de paladar e olfato; 33,3\% apresentaram cefaleia, garganta inflamada e fadiga; $16,7 \%$ tiveram tosse e apneia, e 16,7\% foram assintomáticos. Seguindo o questionário com os que testaram positivo para a corona vírus, os atletas foram perguntados sobre o quanto o período pós-covid-19 influenciou na volta aos treinos, 5 (62.5\%) jogadores responderam que influenciou pouco, teve cansaço, mas concluiu o treino, 2 (25\%) relatou que influenciou muito, teve dificuldades devido a fadiga e falta de ar e $1(12,5 \%)$ disse que não influenciou nos treinos, possivelmente por ter sido assintomático.

Sendo perguntados ainda sobre a influência do corona vírus, porém agora em relação ao desempenho e ritmo de jogo, 1(12,5\%) atleta relatou que não influenciou em seu desempenho e ritmo; 2(25\%) disseram que influenciou muito, e até o momento não voltou ao desempenho e ritmo de antes; 4 (50\%) jogadores afirmaram que o COVID-19 influenciou moderadamente, precisando de um período de mais de 1 mês para retomar ao desempenho e ritmo; 3 (37,5\%) dos outros atletas relataram que influenciou pouco, precisando de um período de menos de 1 mês para retomar o desempenho e ritmo de antes do contagio com o vírus. Por fim, os atletas profissionais contagiados foram questionados se tiveram lesão ao retorno das atividades após o período de 15 dias de quarentena, 2 (25\%) dos 8 atletas sofreram lesões, no qual um teve estiramento dos músculos isquiotibiais e outro rompimento de ligamento cruzado anterior do joelho direito.

\section{Discussão}

Os principais achados do estudo, mostraram que a associação entre o período de isolamento social somado as repercussões provocadas no sistema corporal desses atletas, influenciaram diretamente no desempenho físico para o retorno a suas atividades profissionais. Além disso, mesmo em um curto período de pausa de quinze dias e com permanência dos treinos em casa, as ocorrências de lesões graves responsáveis pelo afastamento de semanas a meses foram encontradas nesse grupo. No estudo em apreço, as médias de idade obtidas foram de 23,23 $\pm 5,41$ anos, idades que se correlacionam de forma bem evidente com os estudos de Silva et al, que obtiveram média de idade de 23,73 \pm 8,63 anos em atletas da seleção voleibol do 
estado do Ceará, evidenciando por uma amostra de jovens promissores, motivados para ter seu lugar e reconhecimento no esporte (Lollgen et al, 2020).

Dos 37 atletas de alto rendimento, 35 (94,6\%) deles responderam que treinaram de alguma forma adaptativa em suas residências no período de isolamento social, porém, mesmo com o treinamento "domiciliar" 62,2\% dos jogadores afirmaram que retornou um pouco fora de ritmo e com condicionamento físico baixo. Contudo, Brett et al, relatou em seu estudo a necessidade de continuar com os treinamentos em casa, pois contribui para amortizar a possibilidade do desenvolvimento da forma grave do COVID-19, além da importância de minimizar os efeitos adversos da interrupção do treinamento em seu retorno as atividades (Corsini et al, 2020; Nascimento et al, 2017).

Ademais, foi avaliado que após a liberação para o retorno das atividades dos clubes e das federações $35,1 \%$ dos atletas se lesionaram, dentre elas, a distensão muscular, foi a mais acometida, correspondendo a 30,7\%, seguido do rompimento do ligamento cruzado anterior em $23,1 \%$ dos jogadores. Nos estudos de Nascimento et al, corroborou com os achados do presente estudo, identificando maior incidência de lesões musculares nos atletas de futebol, com a ocorrências de 59,3\% dos casos. Ekstrand, et al concluíram que o alto risco de distensão muscular reflete a alta intensidade do futebol profissional moderno. Em relação ao diagnóstico confirmado de COVID-19, somente 8 (21,6\%) jogadores foram infectados, destes, os sintomas mais apresentados da amostra com 50\% foram febres, ausência de paladar e olfato; seguido de 33,3\% dos sintomas de cefaleia, garganta inflamada e fadiga; $16,7 \%$ tiveram tosse e apneia, corroborando com os estudos de Ahn, et al.

Com isso, dos atletas infectados, $62.5 \%$ dos jogadores responderam que o COVID-19 teve pouca influência em seu retorno, mesmo assim, o atleta apresentou cansaço, mas concluiu o treino, 25\% relataram que influenciou muito em seu retorno, teve dificuldades devido a fadiga e falta de ar. Além disto, 50\% jogadores afirmaram que o COVID-19 influenciou moderadamente, precisando de um período de mais de 1 mês para retomar ao desempenho e ritmo de antes. Corroborando assim, com o estudo de Hussin et al, que evidencia os efeitos adversos, sobre seu trato respiratório e capacidade aeróbia em curto e longo prazo, e suas variâncias de organismo para organismo, daqueles que foram vítimas da corona vírus (Bushman et al, 2016; Dias et al, 2016). Por fim, os atletas ao retornar do período de 15 dias de quarentena pós contágio com o COVID-19, somente $25 \%$ relataram que sofreram lesões, não correlacionando com o estudo de Dias et al, no qual os atletas que não participaram da pré-temporada possuíram altos níveis de lesão, evidenciando que mesmo em um período curto ausente pode influenciar ao risco de lesões (Wilson et al, 2020; Inciard et al, 2020).

\section{Conclusão}

Diante do exposto, observamos que mesmo os atletas tendo dedicação, empenho e responsabilidade para a continuidade dos treinos de forma adaptativa e isolada em seus lares, retornaram as atividades e treinos com baixo condicionamento físico após o período de isolamento total e pós-covid-19. Contudo, o presente estudo não revelou evidências causais sobre o impacto do isolamento social e do contágio do COVID-19 quando relacionado com o número ou risco de lesões.

Considerando a importância do tema da pesquisa, sugerem-se novos estudos para um maior aprofundamento, visto que a amostra estudada foi pequena e escassa em números de artigos que correlacionem essa "nova" patologia do COVID-19 com o condicionamento físico, desempenho, ritmo e lesões. Além, de um acompanhamento a longo prazo para que sejam observadas as alterações que o COVID-19 proporcionou aos corpos desses atletas. 


\section{Referências}

Ahn, D. G., Shin, H. J., Kim, M. H., Lee, S., Kim, H. S., Myoung, J., Kim, B. T., \& Kim, S. J. (2020). Current Status of Epidemiology, Diagnosis, Therapeutics, and Vaccines for Novel Coronavirus Disease 2019 (COVID-19). Journal of microbiology and biotechnology, 30(3), 313-324. https://doi.org/10.4014/jmb.2003.03011

Bai, Y., Yao, L., Wei, T., Tian, F., Jin, D. Y., Chen, L., \& Wang, M. (2020). Presumed Asymptomatic Carrier Transmission of COVID-19. JAMA, 323(14), 1406-1407. https://doi.org/10.1001/jama.2020.2565.

Barker-Davies, R. M., O'Sullivan, O., Senaratne, K., Baker, P., Cranley, M., Dharm-Datta, S., Ellis, H., Goodall, D., Gough, M., Lewis, S., Norman, J., Papadopoulou, T., Roscoe, D., Sherwood, D., Turner, P., Walker, T., Mistlin, A., Phillip, R., Nicol, A. M., Bennett, A. N., ... Bahadur, S. (2020). The Stanford Hall consensus statement for post-COVID-19 rehabilitation. British journal of sports medicine, 54(16), 949-959. https://doi.org/10.1136/bjsports2020-102596.

Bogoch, I. I., Watts, A., Thomas-Bachli, A., Huber, C., Kraemer, M.U. G., \& Khan, K (2020). Pneumonia of unknown aetiology in Wuhan, China: potential for international spread via commercial air travel. Journal of travel medicine, 27 (2): 1-7. 10.1093/jtm/taaa008

Brooks, S. K., Webster, R. K., Smith, L. E., Woodland, L., Wessely, S., \& Greenberg, N. (2020). The psychological impacto of quarantine and how to reduce it: rapid review of the evidence. The Lancet, 30(395), 912-20. https://doi.org/10.1016/S0140-6736(20)30460-8

Bushman, T. T., Grier, T. L., Canham-Chervak, M., Anderson, M. K., North, W. J., \& Jones, B. H. (2016). The Functional Movement Screen and Injury Risk: Association and Predictive Value in Active Men. The American journal of sports medicine, 44(2), 297-304. https://doi.org/10.1177/0363546515614815

Carmody, S., Murray, A., Borodina, M., Gouttebarge, V., \& Massey, A. (2020). When can professional sport recommence safely during the COVID-19 pandemic? Risk assessment and factors to consider. British journal of sports medicine, 54(16), 946-948. https://doi.org/10.1136/bjsports-2020-102539

Corsini, A., Bisciotti, G. N., Eirale, C., \& Volpi, P. (2020). Football cannot restart soon during the COVID-19 emergency! A critical perspective from the Italian experience and a call for action. British journal of sports medicine, 54(20), 1186-1187. https://doi.org/10.1136/bjsports-2020-102306

Dias, R.G., Gonelli, P.R, \& Cesar, M.C. (2016). Efeito da pré-temporada no desempenho de atletas de futebol feminino. Brasil Medicina e Esporte. 22(2)138141.https://doi.org/10.1590/1517-869220162202147189

Ekstrand, J., Hägglund, M., \& Waldén, M. (2011). Injury incidence and injury patterns in professional football: the UEFA injury study. British journal of sports medicine, 45(7), 553-558. https://doi.org/10.1136/bjsm.2009.060582

Estrela, C. (2018). Metodologia Científica: Ciência, Ensino, Pesquisa. Editora Artes Médicas.

Guan, W. J., Ni, Z. Y., Hu, Y., Liang, W. H., Ou, C. Q., He, J. X., Liu, L., Shan, H., Lei, C. L., Hui, D., Du, B., Li, L. J., Zeng, G., Yuen, K. Y., Chen, R. C., Tang, C. L., Wang, T., Chen, P. Y., Xiang, J., Li, S. Y., \& China Medical Treatment Expert Group for Covid-19 (2020). Clinical Characteristics of Coronavirus Disease 2019 in China. The New England journal of medicine, 382(18), 1708-1720. https://doi.org/10.1056/NEJMoa2002032

Heffernan, K. S., \& Jae, S. Y. (2020). Exercise as medicine for COVID-19: An ACE in the hole?. Medical hypotheses, 142 , 109835. https://doi.org/10.1016/j.mehy.2020.109835

Inciardi, R. M., Lupi, L., Zaccone, G., Italia, L., Raffo, M., Tomasoni, M. D., Dario, S., Cani, M. D., Cerini, M. M. D., Farina, D., Gavazzi, E., Maroldi, R., Adamo, M., Ammirati, E., Sinagra, G., Lombardi, C., Metra, M. (2020). Cardiac involvement in a patient with coronavirus disease 2019 (COVID-19). JAMA Cardiology. 5(7):819-824. doi:10.1001/jamacardio.2020.1096.

Lei, S., Jiang, F., Su, W., Chen, C., Chen, J., Mei, W., Zhan, L. Y., Jia, Y., Zhang, L., Liu, D., Xia, Z. Y., \& Xia, Z. (2020). Clinical characteristics and outcomes of patients undergoing surgeries during the incubation period of COVID-19 infection. EClinicalMedicine, 21, 100331. https://doi.org/10.1016/j.eclinm.2020.100331

Löllgen, H., Bachl, N., Papadopoulou, T., Shafik, A., Holloway, G., Vonbank, K., Jones, NE, Bigard, X., Niederseer, D., Meyer, J., Muniz -Pardos, B., Debruyne, A., Zupet, P., Steinacker, JM, Wolfarth, B., Bilzon, J., Ionescu, A., Dohi, M., Swart, J., Badtieva, V., ... Pitsiladis, YP (2020). Recomendações para o retorno ao esporte durante a pandemia de SARS-CoV-2. BMJ Open Sport \& Exercise Medicine, 6 (1), e000858. https://doi.org/10.1136/bmjsem-2020000858

Nahshon, C., Bitterman, A., Haddad, R., Hazzan, D., \& Lavie, O. (2020). Hazardous Postoperative Outcomes of Unexpected COVID-19 Infected Patients: A Call for Global Consideration of Sampling all Asymptomatic Patients Before Surgical Treatment. World journal of surgery,44(8), $2477-2481$. https://doi.org/10.1007/s00268-020-05575-2

Nascimento, N. A., \& Silva, B. G. d. M. e. (2017). Análise do perfil epidemiológico de lesões esportivas em atletas de futebol profissional. RBFF - Revista Brasileira de Futsal e Futebol, 9(34).

Pereira A. S. et al. (2018). Metodologia da pesquisa científica. UFSM.

Prata, L. O., Rodrigues, C. R., Martins, J. M., Vasconcelos, P. C., Oliveira, F. M., Ferreira, A. J., Rodrigues-Machado, M. D., \& Caliari, M. V. (2017). Original Research: ACE2 activator associated with physical exercise potentiates the reduction of pulmonary fibrosis. Experimental biology and medicine (Maywood, N.J.), 242(1), 8-21. https://doi.org/10.1177/1535370216665174

Rothan, H. A., \& Byrareddy, S. N. (2020). The epidemiology and pathogenesis of coronavirus disease (COVID-19) outbreak. Journal of autoimmunity, 109, 102433. https://doi.org/10.1016/j.jaut.2020.102433

Sepehrinezhad, A., Shahbazi, A., \& Negah, S. S. (2020). COVID-19 virus may have neuroinvasive potential and cause neurological complications: a perspective review. Journal of neurovirology, 26(3), 324-329. https://doi.org/10.1007/s13365-020-00851-2 
Research, Society and Development, v. 11, n. 3, e47711326409, 2022

(CC BY 4.0) | ISSN 2525-3409 | DOI: http://dx.doi.org/10.33448/rsd-v11i3.26409

Silva, H. G. N, Santos, L. E. S., \& Oliveira, A. K. S. Efeitos da pandemia no novo Coronavírus na saúde mental de indivíduos e coletividades. (2020). Journal Nurse Health. 10(4)1-10. https://doi.org/10.15210/jonah.v10i4.18677

Silva, K.M, Almeida, T.G., \& Regis, J.F. Avaliação Funcional do Movimento em Atletas de duas Seleções de Voleibol da Região do Vale do Jaguaribe/Ce. (2017). BIUS Boletim informativo unimotrisaúde em sociogerontologia, 1(8)3-27.

Souza, B. R. G., Teixeira, D. B., \& Sabino, G. S. (2017). Aplicação da avaliação funcional de movimento (FMS) em praticantes de muay thai de belo horizonte/MG. Interdisciplinar ciências. 1(1):51-61.

Toresdahl, B. G., \& Asif, I. M. (2020). Coronavirus Disease 2019 (COVID-19): Considerations for the Competitive Athlete. Sports health, 12(3), 221-224. https://doi.org/10.1177/1941738120918876

Wilson, M. G., Hull, J. H., Rogers, J., Pollock, N., Dodd, M., Haines, J., Harris, S., Loosemore, M., Malhotra, A., Pieles, G., Shah, A., Taylor, L., Vyas, A., Haddad, F. S., \& Sharma, S. (2020). Cardiorespiratory considerations for return-to-play in elite athletes after COVID-19 infection: a practical guide for sport and exercise medicine physicians. British journal of sports medicine, 54(19), 1157-1161. https://doi.org/10.1136/bjsports-2020-102710

Yu, B., Li, C., Chen, P., Zhou, N., Wang, L., Li, J., Jiang, H., \& Wang, D. W. (2020). Low dose of hydroxychloroquine reduces fatality of critically ill patients with COVID-19. Science China. Life sciences, 63(10), 1515-1521. https://doi.org/10.1007/s11427-020-1732-2 